\title{
HACIA UN SISTEMA DE AYUDA \\ A LA DECISIÓN ESPACIAL PARA LA LOCALIZACIÓN DE EQUIPAMIENTOS
}

\author{
POR \\ JOAQUÍN BOSQUE SENDRA, MONTSERRAT GÓMEZ DELGADO, \\ ANTONIO MORENO JIMÉNEZ Y FRANCESCO DAL POZZO
}

\section{INTRODUCCIÓN}

El objetivo de este artículo es describir el posible contenido y organización de un Sistema de Ayuda a la Decisión Espacial (SADE) (Spatial Decision Support System, SDSS), basado en un conjunto integrado de programas informáticos, que permita auxiliar a la determinación de la localización óptima de diferentes tipos de equipamientos: a) los que producen externalidades positivas en su entorno y, por lo tanto, son atractivos para la población, tanto los de carácter público (escuelas, guarderías, hospitales, etc.), como los comerciales (supermercados, hipermercados, grandes almacenes, etc.); b) los centros de distribución comercial dedicados a proporcionar productos a comercios y otros establecimientos; $c$ ) las instalaciones que generan externalidades negativas en su entorno, por lo que son rechazados por la población: vertederos de

J. Bosque Sendra. Departamento de Geografía. Univesidad de Alcalá. Financiación parcial de su actividad por el proyecto de investigación AMB94-1017 de la Comisión Interministerial de Investigación científico y técnica (CUCYT) y el proyecto de investigación 06/0049/98 de la Comunidad de Madrid.

M. Gómez Delgado. Departamento de Geografía, Universidad de Alcalá.

A. Moreno Jiménez. Departamento de Geografía. Universidad autónoma de Madrid.

F. dal Pozzo. Universidad de los Andes. Mérida.Venezuela. 
residuos sólidos urbanos, centros de tratamiento de residuos tóxicos y peligrosos, cárceles, etc.

La estructura de este texto es la siguiente: en el segundo apartado se describe la evolución reciente de los SIG y las principales dificultades para su uso más amplio y difundido. Como solución a algunos de estos problemas el tercer apartado plantea la creación de sistemas de ayuda a la decisión, describiendo su estructura general. En el cuarto apartado se estudia el problema de la localización de equipamientos sociales y las necesidades que suscitan para el uso de información geográfica y, por ello, el interés de la elaboración de un SADE para estos fines. El quinto apartado analiza en detalle las características de un problema de localización y de los distintos tipos de equipamientos e instalaciones a localizar. El sexto apartado describe los conceptos básicos a emplear en la solución de los problemas de localización. El séptimo plantea una posible estructura de un SADE adaptado al tratamiento del problema antes explicado. El octavo apartado da cuenta de algunas realizaciones ya existentes sobre el tema y, finalmente, el último enumera algunas conclusiones.

EL DESARROLLO DE LOS SISTEMAS DE INFORMACIÓN GEOGRÁFICA (SIG) Y SUS APLICACIONES

Los SIG constituyen herramientas muy potentes para el tratamiento de la información geográfica, por ello cada vez se están desarrollando nuevas aplicaciones y utilizaciones en campos y temas diferentes. Muy en concreto los SIG se pueden utilizar, con gran provecho, en la resolución de problemas de ordenación y planeamiento territorial. Para ello, los SIG están dotados de numerosos medios para el análisis de la información geográfica y, sobre todo, para la combinación de diferentes conjuntos de datos, de modo que se obtengan nuevos resultados y mejor información sobre la realidad espacial estudiada. Sin embargo, también es evidente que los SIG presentan algunas dificultades para que se conviertan en la herramienta general de resolución de todo tipo de problemas espaciales, el origen de ello reside en las herramientas demasiado generales y poco especializadas que habitualmente forman parte de la panoplia de medios de un SIG.

La falta de herramientas especializadas es lógica, ya que los SIG son programas de uso general para la descripción y análisis de la in- 
formación geográfica, que no pueden incluir todos los elementos necesarios para tratar la enorme variedad de cuestiones y problemas que cuentan con alguna base geográfica. Para resolver dicha problemática está planteada la posibilidad de construir los llamados sistemas de ayuda a la decisión espacial, que fundamentados en un SIG se adaptan a las necesidades específicas de cada problema.

LOS SISTEMAS DE AYUDA A LA DECISIÓN ESPACIAL (SADE)

Un SADE/SDSS se puede definir como un conjunto de elementos físicos (ordenadores, periféricos, etc.), lógicos (programas, datos...) y procedimientos que facilitan un entorno adecuado para la adopción «racional» de decisiones sobre problemas espaciales.

Un SADE/SDSS como el aquí planteado debe facilitar:

a) La exploración del problema a tratar, con la finalidad de llegar a formular hipótesis que lo resuelvan.

b) La generación de soluciones alternativas abundantes y contrastadas.

c) Una evaluación precisa (a ser posible cuantitativa) de los méritos e inconvenientes de las distintas soluciones.

d) Todas las anteriores actividades se deben de realizar en un entorno de fácil acceso, de manera que los «decisores», que no tienen que ser expertos en el uso de estos programas, puedan utilizarlos con comodidad.

Los SADE tienen definida una estructura general que se puede esquematizar en el gráfico adjunto (véase Fig. 1):

Los componentes de esta estructura se pueden definir así:

Generador de gráficos. Sistema capaz de elaborar gráficos estadísticos y de otro tipo que permitan la exploración de las características del problema. Por lo tanto, capaz de mostrar simultáneamente dos «espacios»: el geográfico y el matemático de las soluciones al problema.

Generador de informes. Capacitado para, por un lado, explorar el problema (usando, por ejemplo, análisis estadístico exploratorio: EDA) $\mathrm{y}$, por otra parte, elaborar informes y resultados. 
ESTRUCTURA DE UN SISTEMA DE AYUDA A LA DECISIÓN

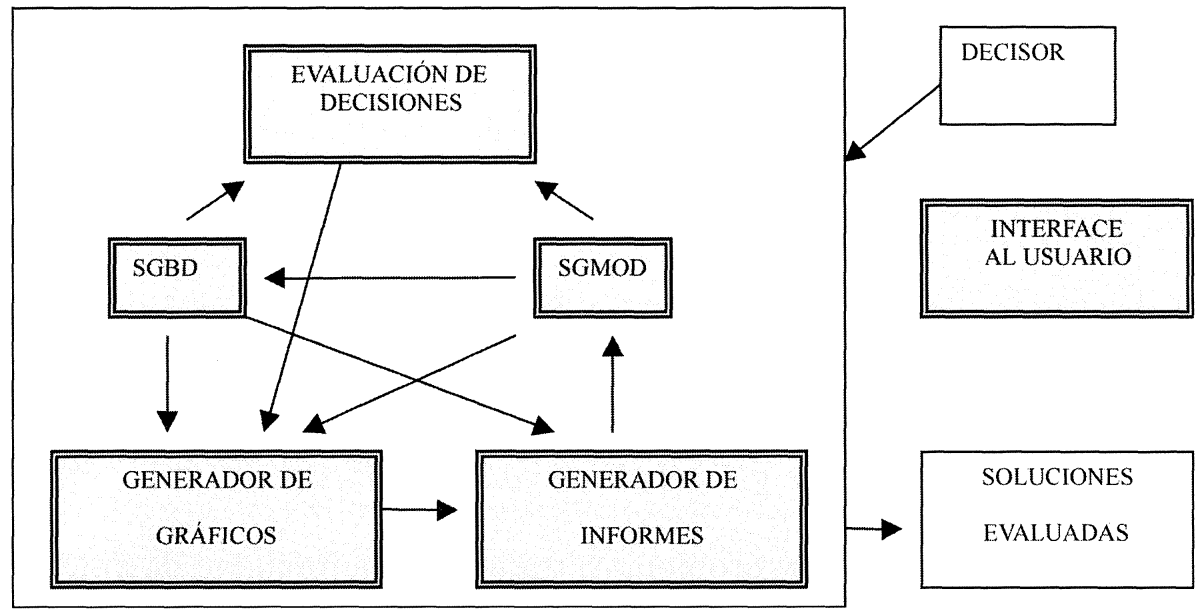

$S G B D:$ Sistema e gestión de bases de datos.

$S G M O D$ : Sistema de gestión de modelos matemáticos.

FigurA 1.-Un esquema de los componentes típicos de un sistema de apoyo a las decisiones (basado en Armstrong,

Densham y Rushton, 1986).

$S G D B$. Sistema de gestión de bases de datos. En concreto, tanto de los datos espaciales como de los temáticos asociados a ellos.

Sgmod. Sistema de gestión de modelos matemáticos. Pensados para generar alternativas y soluciones diferentes al problema tratado por el SADE (en nuestro caso se trataría, básicamente, de los denominados modelos de localización-asignación de instalaciones).

Evaluación de alternativas. Un conjunto de procedimientos matemáticos que permitan jerarquizar las diferentes soluciones estudiadas, entre ellas se pueden mencionar las técnicas de evaluación multicriterio (EMC).

Interfase al usuario. Un mecanismo para la comunicación sencilla e intuitiva entre el usuario (un decisor no experto en manejo de programas) y el sistema. Se puede basar en un sistema experto que guíe al usuario a realizar un análisis inicial y rudimentario y a generar un informe. 
La elaboración de Sistemas de este tipo se puede realizar desde diversos enfoques, en este caso, como se verá mas adelante, el planteamiento consiste en partir de un SIG ya existente y ampliar sus capacidades para poder convertirlo en un verdadero SADE.

\section{EL PROBLEMA DE LA LOCALIZACIÓN DE EQUIPAMIENTOS}

La cuestión central de los problemas estudiados en este trabajo es la determinación de las posiciones geográficas más adecuadas para los distintos tipos de equipamientos, un problema locacional muy característico y específico de la Geografía. Diversas técnicas y metodologías geográficas se han planteado para su correcta resolución; posiblemente la más precisa y adecuada para poder considerar, simultáneamente, criterios de eficiencia y de justicia espacial, son los modelos matemáticos de localización-asignación, que permiten buscar las localizaciones más adecuadas para minimizar costos de transporte (o riesgos a la población), a la vez que los distintos lugares geográficos no se ven muy desigualmente afectados. Por lo tanto, el principal instrumento empleado, para resolver el problema locacional antes planteado serán estos modelos, unidos a procedimientos de evaluación multicriterio de las diferentes soluciones encontradas por ellos. Todos estos elementos se integraran con un SIG para dar origen a un verdadero Sistema de Ayuda a la Decisión Espacial.

La determinación de la posición más adecuada para situar diversos tipos de instalaciones es un importante problema geográfico, con significativas repercusiones económicas, sociales y ambientales. Dado que la demanda de servicios y de bienestar ambiental se ha incrementado notoriamente, su satisfacción, a través de equipamientos de diverso tipo, adquiere gran importancia ya que incide sobre la dimensión bienestar/calidad de vida.

En España existen numerosos casos concretos donde la localización de algún tipo de equipamientos constituye un problema importante. Desde la situación de los servicios de emergencia generales (ambulancias, retenes de bomberos, etc.) hasta la localización de escuelas, institutos o centros de salud en los diferentes municipios, pasando por la problemática social y geográfica planteada por la gestión de los residuos de la actividad económica (posición de vertederos, depósitos de segu-

$$
-571-
$$


ridad o plantas de tratamiento físico-químico), cualquiera de estas cuestiones ofrece un amplio campo para las polémicas, los conflictos sociales y las diferencias políticas y entre los diferentes municipios y las distintas administraciones. Por ello, disponer de herramientas que ayuden a resolver mejor la cuestión es de gran interés.

En este contexto cobra sentido la propuesta desarrollada en este artículo cuya meta final es mejorar la metodología de análisis y planificación de equipamientos, dentro del contexto general de la ordenación territorial.

La necesidad de disponer de sistemas de información para ayudar en el proceso de planificación y formulación de políticas ha emergido recientemente con extraordinaria fuerza. Su papel potencial para definir mejor los problemas, para examinar críticamente si la dotación de servicios es eficiente o equitativa, para predecir y simular los impactos de diferentes alternativas de localización, para el seguimiento de los procesos de ejecución, así como para evaluar los logros y la relevancia de las políticas sectoriales ejecutadas, ha abierto un horizonte de demanda de instrumentos operacionales para tal fin (cf. por ejemplo, England, et al., 1985; Moreno y Alvarez, 1997).

La toma de decisiones espaciales (planificar o gestionar) sobre equipamientos puede requerir análisis muy variados, por lo que también lo serán las técnicas aplicables (vid. Moreno, 1995), no obstante realizando una priorización propondríamos que un SADE/SDSS enfocado al problema de la localización de equipamientos debería posibilitar a los usuarios conseguir, entre otros, los siguientes resultados:

- Determinación de las áreas de servicio de los centros de oferta, tanto las potenciales como las realmente efectivas.

- Medir el nivel de uso de cada servicio o equipamiento.

- Seleccionar qué instalaciones deben ser cerradas (por falta de uso) y en qué lugares deberían abrirse nuevos equipamientos.

- La demanda potencial que la población puede ejercer sobre cada instalación.

- Un análisis de los factores sociales, económicos y espaciales que afectan al uso de los servicios y de cada equipamiento en concreto.

- Ayudar a establecer zonas de reserva de suelo para nuevos centros de atención, en relación a las actividades del planeamiento urbano. 
CARACTERÍSTICAS DEL PROBLEMA DE LA LOCALIZACIÓN.

LOS EQUIPAMIENTOS Y SERVICIOS TRATADOS

En general, se trata de equipamientos o de bienes y servicios suministrados en instalaciones situadas en lugares concretos del espacio, la población que los usa debe desplazarse hasta allí para utilizar ese servicio (a veces es el «bien» el que debe moverse para ser suministrado a la población: el servicio de bomberos por ejemplo). El coste de recorrer esta separación entre instalaciones y población resulta de gran importancia en el uso del servicio. Existe, por ejemplo, un valor de separación, el denominado alcance espacial de un servicio o bien, a partir del cual no merece la pena realizar el traslado para usar el servicio, ya que los costes del transporte superan cualquier beneficio que se puede obtener del uso del servicio o bien.

\section{a) Tipos de equipamientos}

Simplificando los hechos es posible diferenciar, tal y como ya hemos indicado, varios tipos de equipamientos:

1. Equipamientos deseados/deseables, producen un efecto beneficioso en su alrededor (generan externalidades positivas): escuelas, hospitales, comercios, etc.

2. Equipamientos no deseables, su existencia produce un efecto perjudicial en sus alrededores (externalidades negativas): vertederos de basuras, centrales nucleares, cárceles, etc.

Dentro de los equipamientos deseables podemos diferenciar aquellos que son de carácter publico, y, por lo tanto, deben ser disfrutados por todas las personas en las mismas condiciones de acceso y con las mismas dificultades/posibilidades. Por otra parte, existen aquellos otros equipamientos que son de propiedad privada y cuya principal finalidad es proporcionar beneficios a los propietarios a través de su utilización por la población (el ejemplo más evidente son los comercios). En cada caso los principios a emplear en su localización y evaluación son diferentes.

En cualquiera de los tres tipos de equipamientos mencionados se pueden definir algunos elementos que son comunes a todos ellos: 
- Demanda, las personas/empresas que desean utilizar el servicio y que se encuentran distribuidas por el territorio.

- Oferta, los lugares donde se sitúan las instalaciones en las que se puede adquirir o usar los servicios.

- Red de transporte: Vías de comunicación entre oferta y demanda.

\section{b) La organización básica del problema (geometría del sistema)}

Es necesario determinar la geometría de los tres elementos mencionados: oferta, demanda, red de transporte, de cara a su más correcta representación digital en el SIG.

La definición de la red de calles/carreteras a utilizar en el SIG depende de cómo se distribuyan espacialmente la oferta y la demanda, es decir, a qué tipo de elementos espaciales se atribuye la oferta/demanda: puntos, líneas o polígonos.

Distribución espacial de la demanda. Los datos demográficos, que formarán la base de cálculo de la demanda potencial de cualquier instalación de oferta, se pueden obtener, en España (y de manera similar en otros países), en los siguientes formatos:

1. Elementos de tipo poligonal de tamaño relativamente importante en relación a la superficie total del área de estudio: secciones censales, distritos, municipios, etc.

2. Manzanas urbanas (zonas poligonales de tamaño mucho mas reducido).

3. Direcciones postales, existe la posibilidad de geocodificar cada dirección postal como un punto distribuido a lo largo del tramo de calle que le corresponde, de modo similar a como lo realizan diversos SIG del mercado. Un problema importante es que se genera una enorme cantidad de puntos diferentes lo que multiplica el trabajo de cálculo de los modelos de localización-asignación.

En todos estos casos la demanda se puede referir a un punto concreto (centroide de cada zona espacial). Con ello se pierde bastante detalle espacial de la posición de la demanda, pero para muchos estudios resulta suficiente la resolución espacial conseguida y, por otro lado, agi- 
liza los procedimientos de obtención de distancias entre oferta y demanda.

Distribución espacial de la oferta. En principio, la oferta siempre estará ligada a puntos concretos de la región, los lugares concretos donde se ofrecen los diferentes servicios y bienes.

En resumen, tanto la demanda como la oferta quedan, en este planteamiento, asignadas a localizaciones espaciales puntuales, que son las más sencillas de trabajar y las que más intuitivas resultan. Una cuestión posterior es cómo relacionar cada uno de estos puntos con los elementos de la red de calles/carreteras.

Relación entre puntos de oferta/demanda y la red de transporte. Una solución, quizás la más correcta, para relacionar puntos (de oferta y demanda) y red de transporte es añadir nuevos elementos lineales (tramos) a la red, de modo que comuniquen cada punto de oferta/demanda (que no esté ya situado en un nodo de la red) con los nodos de la red que le rodean y están cerca de ese punto. Una solución posible sería trazar en torno a cada punto de oferta/demanda un círculo (de radio prefijado por el usuario, que podría ser proporcional a la superficie de la zona a la que representa ese centroide) y comunicar, con nuevos tramos, el centroide con todos los nodos de la red que estén dentro del circulo mencionado. El principal problema es la gran cantidad de nuevos tramos que se añaden a la red, tramos que, por otra parte, tienen una condición especial: por ellos solo pueden discurrir los movimientos procedentes del centroide original, pero los flujos de otros orígenes no pueden pasar por estos tramos. Todo esto complica mucho utilizar esta solución por lo que no parece la más adecuada.

Un planteamiento ligeramente diferente sería que se obtuviese la distancia más corta entre cada punto de demanda/oferta y los tramos rectos de la red, añadiendo a continuación otro tramo recto que relaciona el punto con esa localización de la red. El resultado final sería el trazado de una red mas completa y detallada, quizás en exceso y por ello difícil de manejar y tratar.

Finalmente, otra posibilidad, la más sencilla de organizar, es asignar cada punto de oferta/demanda a uno de los nodos de la red existente, justamente aquel que se encuentre a menos distancia en línea de recta de él. Esta opción añade algún error al planteamiento, pero resulta muy sen- 
cilla y fácil de utilizar. En diversos programas SIG comerciales existen órdenes que llevan a cabo esta actividad.

En resumen: la oferta y demanda se representan como puntos geométricos con atributos: tamaño de la oferta, tamaño de la demanda, calidad de la oferta, horario de apertura, horario disponible para el uso de la oferta, alcance espacial de un bien. Por su parte, las vías de comunicación son líneas con atributos: velocidad de paso, costo de atravesar cada tramo (pesetas, tiempo, etc.).

\section{CONCEPTOS FUNDAMENTALES DE LA LOCALIZACIÓN DE EQUIPAMIENTOS}

Los procedimientos que se integran en el SADE propuesto parten, generalmente, de un dato dado y fundamental: la distribución espacial de la demanda y de su tamaño. En función de este dato básico y, en ocasiones, de otras informaciones, se deben resolver dos grandes conjuntos de cuestiones. En primer lugar es necesario evaluar la situación de la oferta disponible en un caso real y, por otro lado, buscar nuevas localizaciones para los centros de oferta.

Los elementos que inciden en estas cuestiones son: a) el número de instalaciones existentes o a localizar; b) la posición geográfica de cada una de las instalaciones y c) el valor de la oferta (e.g. atractivo, tamaño) existente en cada uno de los puntos de oferta.

El problema es, por tanto, complicado pues variando cualquiera de los tres factores anteriores se puede mejorar/empeorar la situación de la demanda.

Las tareas a resolver son de diversos tipos:

1. Asignar cada punto de demanda a un centro de oferta o equipamiento.

2. Localizar nuevos equipamientos.

3. Establecer el tamaño de cada equipamiento.

Variando o manteniendo fijos algunos de los elementos antes mencionados se pueden plantear varios tipos de problemas de localización-asignación.

En función de las distintas situaciones usuales es posible definir dos tipos de problemas de localización-asignación: 
A) Están fijos (definidos y prefijados por el usuario) el número y la posición de los centros de oferta. Lo único que se debe resolver por el SADE es el tamaño más adecuado de cada equipamiento. Se trata de un problema simple de asignación, únicamente es necesario determinar a qué puntos de demanda sirve cada centro de oferta y su tamaño. Se trata de una versión del conocido problema del transporte en programación lineal.

Solución:

1. Asignar cada punto de demanda al centro de oferta más próximo. Esto se puede realizar de varias formas: a) Cálculo de la distancia euclidiana más corta entre demanda y oferta. b) En una manera más realista considerando los costos de recorrido entre oferta y demanda a través de la red de carreteras, se puede emplear para ello las órdenes de cálculo de costes de recorrido y análisis de redes de los actuales SIG (por ejemplo, la orden COST en el SIG IDRISI).

2. Sumar la demanda asignada a cada centro de oferta. Esa suma es el tamaño de la oferta mínima a colocar en ese punto.

$B$ Está fijo (definido por el usuario) el número de instalaciones, pero no el tamaño de cada una de ellas (o, dicho en otras palabras, no existe limite superior a la oferta que se puede situar en cada centro). Es variable así mismo la posición de los centros de oferta. Problema más clásico de localización.

Solución:

1. Determinar la posición de los centros de oferta que maximicen la eficiencia y/o la justicia espacial. Esta tarea es la que resuelven los modelos de localización-asignación que se indican más adelante.

2. A continuación realizar un problema de asignación para determinar en concreto a que punto de demanda sirve cada uno de los centros de oferta escogidos y, de ese modo, determinar su tamaño concreto.

Es posible enumerar algunos principios básicos a tener en cuenta para resolver las tareas planteadas (McAllister, 1976; Morril y Symons, 1977): 
a) Principio de la eficiencia espacial. Se busca/evalúa positivamente configuraciones de la oferta que, dada una posición concreta de la demanda, determinen un valor mínimo (o máximo según el tipo de equipamientos) de alguna medida (la suma total de movimientos, la distancia máxima entre oferta y demanda, etc.) de los desplazamientos necesarios para utilizar los servicios por toda la población.

b) Principio de la justicia espacial. Para gran número de los equipamientos estudiado, en concreto los deseables de titularidad pública y los no deseables, es importante igualmente, que no existan grandes diferencias geográficas en la posibilidad de usar o ser afectado por los servicios planteados. Las distancias existentes entre demanda y oferta no deberían ser excesivamente diferentes, ya que esto implica grandes diferencias en el acceso a la oferta.

Los dos principios anteriores se tienen en cuenta, de diversas formas, a la hora de establecer los métodos de evaluación de la situación inicial y los modelos de localización-asignación incluidos en el sistema de ayuda a la decisión que se propone a continuación.

\section{LA ESTRUCTURA DE FUNCIONES DE UN SADE PARA LA LOCALIZACIÓN DE EQUIPAMIENTOS}

A continuación se muestra la estructura y la lista de opciones que consideramos se deberían integrar en un SADE con la finalidad de ayudar a resolver la localización de equipamientos sociales de diverso tipo. Todos estos procedimientos se superpondrían a las funciones usuales de entrada de datos, cartografía, análisis y búsqueda selectiva habitualmente existentes en los SIG comerciales al uso.

\section{ÍNDICE GENERAL DE UN SADE PARA EQUIPAMIENTOS SOCIALES}

Funciones utilizables en la exploración de la situación existente con el fin de desarrollar hipótesis para la resolución de su problemática. 
Evaluación de la adecuación de la oferta a la demanda. Equipamientos deseables

a) Medidas globales de la adecuación de la oferta a la demanda. 1. ${ }^{\circ}$ Suma de las distancias existentes entre oferta y demanda; $2 .^{\circ}$ suma ponderada por la demanda de cada punto entre oferta y demanda; $3{ }^{\circ}$ distancia máxima y distancia mínima entre oferta y demanda, y $4 .^{\circ}$ desviación típica de las distancias.

b) Evaluación de la situación en cada zona de servicio. Cálculo de los anteriores valores pero ahora referidos a cada zona de servicio de cada uno de los centros de oferta existentes (a cada equipamiento se asignan los puntos de demanda que están a menor distancia de él que de cualquier otro).

c) Población servida y no servida por la oferta dada su configuración espacial y un valor del alcance espacial. Fijado un valor de distancia, la que se corresponde, por ejemplo, al alcance espacial de un servicio, se determina los puntos de demanda que se encuentran a una distancia menor que la prefijada de un centro de oferta y se suma la población residente en ellos. Ésta es la población servida.

d) Cartografía de los puntos de demanda y de oferta. En el artículo de Amstrong y otros (1992) se puede encontrar una enumeración de los diferentes tipos de mapas útiles para el tratamiento de los problemas de localización. En concreto, dos de ellos son de gran interés: el mapa tipo «araña» y el mapa «anular». El mapa tipo «araña» o de «líneas de deseo» (spider ó desire lines maps en inglés), relaciona con líneas los puntos de oferta y los puntos de demanda asignados a cada uno de ellos. El mapa anular muestra mediante un circulo, de radio prefijado, en torno a los puntos de oferta o a los puntos de demanda las zonas peor y mejor servidas por la oferta.

\section{Equipamientos no deseables}

a) Medidas globales de la adecuación de la oferta a la demanda. Similares a las planteadas en el apartado A anterior.

b) Distancia minima entre cada punto de demanda (población) y un punto de oferta. Constituye una medida simple, pero efec- 
tiva, de la posible incidencia de las instalaciones no deseables sobre cada punto poblado.

c) Población afectada y no afectada por la oferta dada su configuración espacial y un valor del alcance espacial. Se trata de un análisis similar al del apartado $\mathrm{C}$ precedente.

d) Cartografía de la influencia de los puntos de demanda y de oferta. Usando de manera similar los mapas citados en el apartado D previo.

Otra posibilidad de interés sería el análisis interactivo de las localizaciones existentes, en modo semejante a como lo plantea Densham (1996). Aunque esto exige disponer de medios de proceso más potentes de lo que son los actuales microordenadores.

Medidas de la accesibilidad de la demanda.

Instalaciones deseables

a) Accesibilidad obtenida considerando la distancia (coste de recorrido) en cada punto de demanda al centro de oferta más próximo. Determinación de la distancia recta (o el coste de recorrido a través de la red de comunicaciones) más corta existente entre cada punto de demanda y un centro de oferta. Realización de un mapa que muestre cómo varía ese valor en los distintos lugares donde existe demanda.

b) Accesibilidad (en cada punto del mapa) obtenida considerando la distancia (coste de recorrido) en cada punto del mapa al centro de oferta más próximo. De manera similar al caso anterior obtención de la distancia en línea recta (o del coste de recorrido) más corto que existe entre cada punto del territorio (tenga o no demanda) y un centro de oferta. Elaboración de un mapa con estos valores, el cual permite determinar, en una primera aproximación, los lugares más adecuados para situar nuevas instalaciones (los que presenten peores niveles de accesibilidad a la oferta ya existente).

c) Accesibilidad obtenida considerando la distancia a los centros de oferta y tamaño de la oferta en ellos. Se trata de una medida de la accesibilidad donde interviene no solo la distancia 
sino además el tamaño de la oferta existente en cada centro de este tipo. Existen varias fórmulas para su obtención, una de las más empleadas es la del potencial de población (demanda) sobre cada equipamiento (expresan las diferencias de accesibilidad entre ellos).

1. Fórmula del potencial con descenso lineal de la acción de la distancia (Brocker, 1989; Jones, 1981; Oberg, 1976).

$$
A C_{i}=\Sigma_{j}\left(\text { Oferta }_{i}-\text { Oferta }_{j} \cdot \frac{D_{i j}}{R}\right)
$$

donde $A C_{i}$ es la accesibilidad en el punto i; Oferta $\mathrm{j}_{\mathrm{j}}$ oferta del bien/servicio existente en el punto $j ; D_{i j}$, distancia entre el punto $i$ y el centro de oferta $j ; R$, alcance espacial del bien o servicio.

2 Fórmula del potencial, con descenso exponencial de la acción de la distancia (Brocker, 1989; Oberg, 1976; Jones, 1981).

$$
A C_{i}=\Sigma_{j}\left(\frac{\text { Oferta }_{j}}{e^{A x D_{i j}}}\right)
$$

donde $A C_{i}$ es la accesibilidad en el punto $i$; Oferta ${ }_{j}$, oferta del bien/servicio existente en el punto $j ; D_{i j}$, distancia entre el punto $i$ y el centro de oferta $j$; A, parámetro a definir de fricción de la distancia (en general debería ser menor de 0.1, en otro caso produce valores muy grandes en el denominador de la función y ésta adopta valores minúsculos).

En los dos casos anteriores se puede considerar, al mismo tiempo, el alcance espacial del servicio/bien estudiado; cualquier punto que se encuentre fuera del alcance espacial (valor seleccionado por el usuario) de todos los centros de oferta recibe una accesibilidad cero. Estas medidas de la accesibilidad consideran en cada punto la acción de todos los centros de oferta existentes en la zona y no únicamente del más próximo a cada punto. 
Un resultado básico de estos dos procedimientos es la obtención de mapas de accesibilidad que valoran la diferente situación de cada punto de demanda.

d) Accesibilidad (en cada punto de demanda) obtenida considerando la relación entre tamaño de la oferta y tamaño de la demanda dentro del alcance espacial del servicio. Conocidas: $1 .^{\circ}$ la oferta total dentro del alcance espacial de un punto de demanda y $2 .^{\circ}$ la demanda dentro del alcance espacial de cada centro de oferta, se puede obtener una razón como la siguiente:

$$
\operatorname{Accesib}_{i}=\frac{\text { Oferta }_{\text {total }_{i}}}{\sum_{j=1}^{j=R} \text { Demanda }_{\text {total }_{j}}},
$$

donde $i$ indica cada uno de los puntos de demanda; $j$, cada uno de los centros de oferta; $R$, es el número de los centros de oferta que están dentro del alcance espacial de un punto de demanda.

Como en los casos anteriores el resultado es un mapa que muestra la variación en los puntos de demanda de esta medida de la accesibilidad.

e) Accesibilidad espacio-temporal en los puntos de demanda. La accesibilidad espacio temporal intenta medir las posibilidades ofrecidas al usuario por la posición espacial de la oferta, considerando el tiempo que se tarda en acceder a ella (viaje), el horario de apertura de los centros de oferta y la duración temporal de su utilización. Los planteamientos teóricos básicos de este enfoque para el análisis de la accesibilidad residen en la denominada Geografía del Tiempo planteada por T. Hagerstrand (Bosque Sendra et al., 1992).

Conceptos básicos: $a$ ) Centro de oferta ACCESIBLE, es decir dentro del alcance espacial (medido en unidades de tiempo) de ese bien. $b$ ) Centro de oferta ABIERTO, dentro del alcance espacial de ese bien y abiertos a una hora concreta del día. $c$ ) Centro de oferta UTILIZABLE, dentro del alcance espacial de ese bien y utilizable (el tiempo que estará abierto ese centro de oferta es mayor que la duración del viaje más la duración del uso del servicio) a una hora del día. 
En una primera aproximación el análisis se puede realizar para todos los centros de demanda existentes: contabilizando cuantos centros de oferta cumplen alguna de las situaciones anteriores en relación a cada punto de demanda. En segundo lugar, se puede llevar a cabo para un punto de demanda escogido por el usuario e identificar los centros de oferta concretos que cumplen alguna de las condiciones anteriores.

f) Accesibilidad espacio-temporal de todos los puntos del mapa. De manera similar al caso anterior, pero aplicado a cada punto del territorio y no sólo a aquéllos que cuentan con una demanda asignada. Nuevamente su principal utilidad es servir de guía para la búsqueda de nuevas localizaciones adecuadas para otros puntos de oferta.

Determinación de áreas de influencia de los centros de oferta. Problemas de asignación

A) En función de la distancia más corta entre oferta y demanda. Aplicar el procedimiento del apartado II.A para determinar los puntos de demanda relacionados con cada centro de oferta. Esta información puede servir para construir el mapa «Araña/Spider» antes mencionado.

B) En función del coste de recorrido a los diferentes centros de oferta y del tamaño de la oferta en cada uno de ellos. Entre los varios modelos existentes en la bibliografía, podría mencionarse el clásico de HUFF (1964), cuyas ideas han sido desarrolladas con posterioridad, según la siguiente ecuación:

$$
\operatorname{Prob}_{i j}=\frac{\frac{\text { Oferta }_{j}}{D_{i j}^{A}}}{\sum \frac{\text { Oferta }_{j}}{D_{i j}^{A}}},
$$

donde $\operatorname{Prob}_{i j}$, es la probabilidad de que la población situada en el punto $i$ utilice el servicio/bien colocado en la posición $j$; Oferta ${ }_{j}$ oferta del bien/servicio situado en $j ; D_{i j}$, distancia entre el punto $i$ el centro de oferta $j$; A, un exponente que mide la fricción ejercida por la distancia a recorrer para hacer uso de ese bien/servicio. 
Procedimientos que permiten llevar a cabo la generación

de soluciones al problema de la localización

óptima de instalaciones.

Modelos de localización-asignación.

Instalaciones deseable

A) Modelo p-mediano (PMEDIANO). Objetivo MINISUM. El objetivo de este modelo es minimizar la suma de los productos de la población o demanda de cada punto y la distancia al centro de oferta más próximo. La ecuación general es la siguiente: Minimizar la función:

$$
F=\Sigma_{i=1}^{n} \Sigma_{j=1}^{m} w_{i} D_{i j}^{b} x_{i j}
$$

donde: $W_{i}$, demanda existente en el punto de demanda $i ; D_{i j}$, es la distancia/coste que separa el punto de demanda $i$ del punto candidato a recibir la oferta $j ; x_{i j}$, vale 1 si el centro de oferta $j$ es el más próximo al punto de demanda $i$, cero en otro caso; $n$, es el numero total de puntos de demanda y $m$ es el numero total de puntos candidatos a considerar (las instalaciones ya existentes, y que se desean conservar, más las nuevas); $i$, punto de demanda; $j$, centro de oferta; $p$, número de instalaciones a localizar; $b$ es un exponente elegido por el usuario, el valor por defecto es 1, el cual equivale al modelo p-mediano clásico. Este esquema matemático se mantiene con ligeras variantes en los restantes modelos, por ello no será repetido en estos, en diversas publicaciones se puede encontrar el detalle matemático de cada caso.

De este modo se alcanza la máxima eficiencia espacial pues el conjunto de traslados que tiene que efectuar la demanda (o la oferta) para surtirse del servicio es el mínimo posible dada la distribución espacial de la demanda y de los puntos candidatos planteados (Bosque y Moreno, 1990; Daskin, 1995; Hillsman, 1984; Moreno y López de los Mozos, 1989). Usando un exponente b mayor de la unidad, se puede alcanzar un cierto grado de justicia espacial en la posición de los nuevos centros de oferta.

B) Modelo p-mediano con restricción de máxima distancia (MEDIRES). Objetivo MINISUM con restricción de alejamiento. El objetivo de este modelo es minimizar la suma de los productos de la po- 
blación o demanda de cada punto y la distancia al centro de oferta más próximo, evitando que ningún elemento de la demanda se encuentre a más de una distancia máxima definida por el usuario.

En este caso, se alcanza la máxima eficiencia espacial pues el conjunto de traslados que tiene que efectuar la demanda (o la oferta) para surtirse del servicio es el mínimo posible dada la distribución espacial de la demanda y de los puntos candidatos planteados, al mismo tiempo ningún elemento de la demanda está demasiado alejado de un punto de oferta y el grado de injusticia espacial se hace menor.

El modelo busca que ningún elemento de la demanda pueda estar situado a mayor distancia de un centro de oferta que el alcance espacial de ese servicio (la distancia máxima fijada por el usuario del modelo), cuidando, simultáneamente, la eficiencia espacial. Esta solución a veces no es posible con el número de instalaciones planteado por el usuario, en esos casos es necesario que el programa advierta al usuario de esta circunstancia para que se cambie alguna de las condiciones del caso, generalmente aumentando el número de instalaciones a localizar (Bosque Sendra y Moreno Jiménez, 1990).

C) Modelo de cobertura máxima (COBEMAX). Objetivo de máxima cobertura: El objetivo de este modelo es maximizar la cantidad de demanda que se encuentra dentro de una distancia $\mathrm{R}$ (prefijada por el usuario) de algún centro de oferta.

La idea básica es el establecimiento de un radio de cobertura, $R$ (que podemos hacer equivalente al llamado alcance espacial de un bien o servicio), este radio muestra la distancia que es razonable recorrer para usar el servicio, por encima de este valor el número de personas que se moverán para ir a utilizar este servicio será muy reducido. El objetivo, por lo tanto, es situar los nuevos centros de oferta de modo que la mayoría o la totalidad de la demanda se encuentre a menos de este valor de distancia de un centro de oferta (Bosque Sendra y Moreno Jiménez, 1990).

D) Objetivo de máxima cobertura con restricción de alejamiento (COBERES). El objetivo de este modelo es maximizar la cantidad de demanda que se encuentra dentro de una distancia $R$ (prefijada por el usuario) de un centro de oferta, controlando que la demanda se encuentre en su totalidad dentro de un rango $\mathrm{S}$ (mayor que $\mathrm{R}$ ). 
Como en el modelo anterior, el concepto fundamental de esta formulación es el establecimiento de un radio de cobertura, $R$ (que podemos hacer equivalente al llamado alcance espacial de un bien o servicio), este radio muestra la distancia que es razonable recorrer para usar el servicio. El objetivo, por lo tanto, es situar los nuevos centros de oferta de modo que la mayoría o la totalidad de la demanda se encuentre a menos de este valor de distancia de un centro de oferta. Además toda la demanda debe encontrarse a menos de la distancia máxima de alejamiento admisible (S).

El objetivo es intentar adecuar la eficiencia con la justicia espacial, para ello el modelo busca las localizaciones de la oferta de modo que la mayoría de la demanda se encuentre dentro de una distancia fijada por el usuario (normalmente será el alcance espacial del bien/servicio estudiado) y al mismo tiempo se intenta atender a la eficiencia espacial (valor mínimo de la distancia total recorrida) de las localizaciones (Bosque Sendra y Moreno Jiménez, 1990).

E) Modelo p-mediano con restricción de horarios de apertura. El objetivo de este modelo de localización-asignación es determinar la posición geográfica más adecuada y el horario de apertura óptimo para facilitar el uso de un conjunto de equipamientos, conocidos la posición de la demanda y los horarios de uso potencial de este tipo de equipamientos (Bloxham y Church, 1991).

F) Modelo p-center. En este caso el objetivo es minimizar la suma de las distancias ponderadas entre cada punto de demanda y el equipamiento más alejado de él.

Lo que se pretende alcanzar es la máxima eficacia espacial considerando específicamente la distancia que separa a cada punto de demanda respecto al equipamiento más lejano, de este modo se asegura que la máxima tardanza en suministrar el servicio (el tiempo de retraso se considera directamente proporcional a la distancia) se hace mínima. Por ello, este modelo es más adecuado para estudiar la solución en el caso de los servicios de emergencia (bomberos, ambulancias, policía, etc.), donde el tiempo máximo de retraso es muy importante (Pelegrin, 1996).

Los modelos hasta ahora mencionados son únicamente una muestra de todos los que se han ido desarrollando en esta línea, pero constituyen

$$
-586-
$$


los más usuales y conocidos y, por lo tanto, los más recomendables para su introducción en un sistema de ayuda a la decisión como el aquí planteado.

Instalaciones no deseables

A) Objetivo MAXISUM. El objetivo de este modelo es maximizar la suma de los productos de la población o demanda de cada punto y la distancia al centro de oferta más próximo, es decir las distancias mínimas entre punto con población e instalación. La formulación que se plantea es la inversa del MINISUM, de modo que es fácilmente tratable con las mismas herramientas que éste (Muñoz Pérez, 1996).

De este modo se alcanza la máxima eficiencia espacial desde el punto de vista de la población afectada, ya que la separación entre instalaciones no deseables y el conjunto de la población es la máxima posible. No obstante, este modelo no considera otra forma de la eficiencia espacial para este problema, la referente a los productores de residuos o sustancias a ser tratadas en estas instalaciones, para ellos la distancia total a recorrer puede ser, dada la solución encontrada por este modelo, extraordinariamente grande y por ello, la solución, inadecuada o imposible. La consideración de los dos tipos de eficiencia requiere un planteamiento diferente del problema (Bosque y Franco, 1995).

B) Objetivo de cobertura mínima. El objetivo de este modelo es minimizar la cantidad de personas que están a menos de una distancia $\mathrm{R}$ (prefijada por el usuario) de una instalación no deseable. La formulación planteada es la inversa del objetivo de máxima cobertura, de modo que es fácilmente tratable con las mismas herramientas que éste.

Como en el caso del modelo de cobertura máxima, la idea básica es el establecimiento de un radio de cobertura (que podemos hacer equivalente a la distancia por debajo de la cual la instalación es peligrosa o produce molestias), este radio muestra la distancia que es razonable separe, como mínimo, la población de la instalación más próxima, por debajo de este valor el número de personas debería ser el mínimo posible. El objetivo, por lo tanto, es situar las nuevas instalaciones no deseables de modo que la mayoría o la totalidad de la población se encuentre a más distancia de este valor de una instalación (Bosque y Franco, 1995). 
C) Modelo Maximin. La finalidad del modelo Maximin es la maximización de la distancia mínima entre las instalaciones y el centro poblado más próximo (Bosque y Franco, 1995; Erkut y Neuman, 1988; Muñoz Pérez, 1996).

Este modelo plantea el concepto de máxima eficiencia espacial de modo diferente a los anteriores, ahora se trata de asegurar que la distancia más corta entre un centro no deseable y alguno de los lugares poblados se haga lo más grande que sea posible (y de este modo las molestias se conviertan en las mínimas posibles), dada la distribución espacial de los lugares poblados y de los puntos candidatos planteados.

Únicamente se han planteado algunos de los modelos de este tipo, los más conocidos y usuales. Existen muchos otros, algunos más complejos, pero, igualmente interesantes, por ejemplo Ratick y White (1988). No obstante, con los aquí mencionados se podrían resolver muchos problemas de este tipo.

\section{Instalaciones comerciales}

A) Modelo maximización de la asistencia. Objetivo de maximización de la demanda con elasticidad lineal respecto a la demanda (DECLIN). El objetivo de este modelo es asegurar la máxima cantidad de usuarios a los centros de oferta, para ello se supone que existe una disminución de los usuarios que se dirigen a un centro de oferta de manera directamente (linealmente) proporcional al aumento de la distancia que separa un punto de demanda y del centro de oferta más próximo.

Como ya se ha indicado la posición de los centros candidatos busca asegurar la llegada del máximo número de usuarios a los centros de oferta, de modo que la demanda tenga que recorrer la mínima cantidad posible de distancia. Para ello se privilegian las zonas donde se concentran los puntos con mayor cantidad de demanda. En Bosque Sendra y Moreno Jiménez (1990) se puede encontrar la formulación matemática de este modelo y de muchos de los anteriores.

B) Objetivo de maximización de la demanda con descenso de la demanda según una función exponencial negativa de los costes de transporte (DESNOLIN). De manera similar al caso anterior se tiene en cuenta la incidencia del aumento de la distancia en la disminución de 
la demanda atraída, pero ahora considerando un descenso exponencial de la atracción con el incremento de la distancia (Hodgart, 1978).

Procedimientos que permiten evaluar las soluciones desarrolladas en los apartados anteriores

Evaluación multicriterio de las soluciones encontradas

A) Suma lineal ponderada de las puntuaciones de las distintas soluciones. Mecanismo para evaluar de modo razonable las cualidades de las distintas soluciones encontradas, para ello se emplea el procedimiento de la suma lineal ponderada de las valoraciones establecidas de cada solución en los diferentes criterios que se usen para la evaluación. De este modo, la solución que obtenga mayor puntuación será la solución más adecuada al problema (Barba y Pomerol, 1997; Barredo, 1996).

B) Normalización de las alternativas a una única escala de medida. Los diferentes criterios que se usen para valorar las alternativas pueden estar expresados en unidades y escalas muy diferentes, por ello antes de utilizar el procedimiento de la suma lineal ponderada es necesario normalizar todas las unidades y escalas, para ello se propone incluir varias de las posibilidades de normalización usuales (Barba y Pomerol, 1997).

C) Determinación de pesos de los factores. En el proceso de evaluación de alternativas es necesario ponderar cada uno de los criterios definidos, la obtención de estos pesos o ponderaciones se ve facilitada al disponer de métodos como el propuesto por Saaty para resolver esta cuestión (Barba y Pomerol, 1997; Barredo, 1996).

Características de la interfase de acceso a los procedimientos antes planteados

Una característica muy relevante de los Sistemas de ayuda a la decisión es su facilidad de uso y su organización intuitiva y asequible. Los 
destinatarios de estos instrumentos no suelen ser expertos en la utilización de ordenadores y de programas, en principio son los decisores directos: políticos, ejecutivos y directores de empresa. Por lo tanto, el sistema debe estar preparado para activamente ir guiando al usuario a la realización de los análisis y cálculos que necesite.

Esta circunstancia resulta bastante complicada de resolver, y lo es más en el caso del problema aquí tratado, bastante complejo y difícil de estructurar de modo simple y evidente. La mejor solución sería la elaboración de un sistema experto que permita elegir y realizar los análisis necesarios de manera adecuada y sencilla, pero esto se ve gravemente dificultado por la falta de estructuración del problema que estudiamos.

Ante esta dificultad caben varias posibilidades. En primer lugar, considerar que el usuario típico de este SADE no será un político o ejecutivo con nulos o muy reducidos conocimientos del tema, por el contrario, crear el SADE en función de su empleo por técnicos de las instituciones (Ayuntamientos, Administración regional, etc.) o de las empresas, que, por lo tanto, poseen un mayor conocimiento de la cuestión estudiada y están más preparados para el uso de programas informáticos complejos. En segundo lugar, elaborar un tipo de interfase muy activa (en la línea de los Wizzard del programa Office de Microsoft), de modo que planteen al usuario, en cada momento, las opciones más importantes que el SADE ofrece, ilustrando mediante textos o gráficos en que consisten esas posibilidades.

Un ejemplo de estas opciones podría ser la lista de elecciones que se ofrecen a continuación. Partimos del principio de que la base de datos necesaria para tratar estos problemas ha sido creado previamente, usando para ello el SIG al cual se le han añadido las posibilidades y programas que lo convierten en un SADE de este tipo. Es decir, el usuario tiene disponible información en el formato concreto del SIG que se utiliza de los siguientes aspectos: a) Distribución espacial de la demanda, b) distribución espacial de la oferta existente y de los lugares candidatos a recibir un nuevo lugar de oferta y c) red de comunicaciones que permite relacionar los dos aspectos anteriores.

A partir de estos datos es posible llegar a tratar y a resolver los problemas de localización que nos interesan. Para ello se puede esquematizar las tareas a resolver en el siguiente conjunto de elecciones que podría ir formulando la interfase «activa» que planteamos: 
1 Definir por el usuario los parámetros básicos del problema:

- Valor del alcance espacial del bien o servicio a estudiar.

- Número de instalaciones a localizar (ya sean nuevas o preexistentes que se desean eliminar).

- Tiempo mínimo que es necesario para utilizar el servicio o obtener el bien estudiado.

- Criterios que se van a utilizar para comparar las diversas soluciones encontradas, por ejemplo: eficiencia global de la solución (medida por el tiempo de recorrido total), justicia global (medido por alguno de los indicadores mencionados en el apartado I del índice del SADE), coste de construcción medio de cada nueva instalación, etc.

2. Elegir entre estudiar instalaciones deseables, no deseables y comerciales.

3. Seleccionar si se desea evaluar una situación existente o buscar nuevos puntos para localizar instalaciones.

4. En el caso de la evaluación de una situación la siguiente elección es entre evaluación global de la situación o evaluación detallada de cada punto de demanda o del territorio. En el primer caso se deberán emplear los procedimientos enumerados en el apartado I, de acuerdo con el tipo de instalación (deseable o no deseable), todos estos procedimientos podrían ser llevado a cabo de manera automática por el programa sin nuevas consultas al usuario, presentando a continuación los resultados de los diferentes procedimientos de una forma los mas gráfica y asequible posible. En el caso de una evaluación detallada se usarían los métodos descritos en los apartados II y III. Como en el caso anterior el sistema podría efectuar todos los procedimientos mostrando sus resultados al usuario.

5. En el caso de búsqueda de nuevas localizaciones, el sistema haría uso de los modelos de localización asignación descritos en el apartado IV, eligiendo los métodos adecuados al tipo de instalación indicado previamente. Con la aplicación de las diferentes opciones el sistema obtendría un conjunto de soluciones que podría mostrar al usuario antes de pasar a su evaluación.

6. Las soluciones encontradas serían evaluadas usando los procedimientos antes mencionados, comparándolos con la situación inicial ya conocida. 
7. Por otra parte, haciendo uso de los métodos descritos en el apartado V, se podrá comparar cada una de las soluciones encontradas de modo que el usuario tenga el máximo número posible de criterios para su elección final. Para ello planteará al usuario una matriz de comparación por pares de los distintos criterios enumerados como importantes en la definición de comparaciones de las soluciones y, al mismo tiempo, normalizara todos estos criterios a una misma escala, empleando alguno de los métodos que se han incluido en el SADE.

Un elemento primordial de esta interfase es la presentación del modo más intuitivo y fácil posible del conjunto de resultados que el sistema genera. Con estas ideas, todavía demasiado genéricas, consideramos que sería posible iniciar la creación de un SADE que realmente fuese una herramienta de utilidad para la adopción de mejores decisiones sobre estos temas.

\section{Algoritmos DE CÁlCULO DE LOS MODELOS DE LOCALIZACIÓN ASIGNACIÓN}

Una cuestión importante a la hora de elaborar un Sistema de ayuda a la decisión de este tipo se refiere a los procedimientos a utilizar para el cálculo de los modelos de localización-asignación. En general, no es posible utilizar un procedimiento analítico (óptimo), por ejemplo la programación lineal, para su resolución en casos reales. En esas circunstancias el número de puntos de demanda y el de puntos candidatos a recibir un centro de oferta puede ser importante, por ejemplo, más de 1000 , y es conocido que la programación lineal conllevaría un tiempo de cálculo demasiado extenso para que resultara útil (Church y Sorensen, 1996). Por ello, es necesario emplear procedimientos de búsqueda heurística para solucionar la cuestión (Densham y Rusthon, 1991 y 1992; Moreno Pérez, 1996).

Existen numerosos métodos heurísticos preparados para resolver los modelos de localización-asignación, en el trabajo de Church y Sorensen (1996) se contiene un buen resumen de la literatura sobre el tema y su conclusión es que la elección del algoritmo de Teizt y Bart (el primero de todos los elaborados, en 1968) sigue siendo una de las convenientes, sino la mejor, de las opciones posibles. La otra posibilidad que estos autores 
recomiendan es el procedimiento denominado GRIA. No obstante, el algoritmo de Teizt y Bart resulta muy fácil de programar, ha sido utilizado en numerosas ocasiones y es muy bien conocido (por ejemplo, existen subrutinas que lo describen perfectamente, Goodchild y Noronha, 1983). En resumen, parece adecuado seleccionar este procedimiento para resolver los modelos de localización-asignación que se puedan incluir en un SADE de este tipo.

Una cuestión importante de este procedimiento, o de los otros del tipo heurístico, es la posibilidad de que no se identifique siempre la solución óptima global, sino que el algoritmo localice un mínimo local que no sea el mejor de los posibles. Para evitarlo en alguna medida, se recomienda realizar varias búsquedas con el mismo procedimiento, partiendo en cada ocasión de una configuración inicial de puntos candidatos diferentes, en caso de que cada vez se localice la misma solución podemos estar mas convencidos de su validez global.

El articulo citado de Church y Sorensen (1996) recomienda una regla empírica que ayude a aumentar la probabilidad (nunca la seguridad absoluta) de haber encontrado la solución óptima: «Reinicie la búsqueda de nuevas soluciones hasta que la mejor solución encontrada se repite un número $t$ de veces, en ese momento pare el proceso de búsqueda». Mas aún, los autores consideran que 3 es una buena cifra para el valor de $t$ Una opción podría ser que los programas que resuelven los modelos de localización-asignación trabajen repitiendo las búsquedas (cambiando en cada ocasión de configuración inicial) hasta que la mejor solución se repite tres veces.

Este mecanismo parece útil que sea incluido en el SADE de cara a mejorar la validez de los resultados encontrados por el sistema.

\section{DESARROLLOS YA EXISTENTES DE ESTE TIPO DE PRODUCTOS}

La línea de investigación de los sistemas de apoyo a la decisión cuenta ya con una cierta tradición y prometedores avances en diversos campos, como denotan las publicaciones existentes e incluso la existencia de una revista (Decision Support Systems) centrada en esa temática; no obstante subsisten problemas conceptuales y metodológicos (Er, 1988).

En el campo espacial o geográfico existen algunas embrionarias aportaciones de interés, por ejemplo la de Kohsaka (1993) o la de Ji y 
Johnston (1994). En el sitio WEB: http://ccg.leeds.ac.uk./mce/mcehome.htm es posible acceder a un interesante ejemplo interactivo de SADE preparado para resolver este tipo de problemas de localización de instalaciones, en concreto las no deseables.

Por su parte, Enache (1994) realiza un balance de los problemas todavía existentes para integrar los SIG con los Sistemas de ayuda a la decisión, discutiendo la aportación que puede suponer el usar los Sistemas de ayuda a la decisión ya existentes en el mercado, tales como: a) Sistemas de ayuda a la decisión basados en modelos de elección (models of choice). Tales como los programas: Expert Choice 7.2 y Decision Pad. b) Sistemas de ayuda a la decisión basados en patrones de búsqueda (pattern seeking): Programas KnowledgeSeeker e IXL. Esta es otra posibilidad de construir un SADE: integrar en un SIG programas ya existentes para ayuda a la decisión, aunque las dificultades para incluir datos espaciales en este tipo de software es bastante importante. Se trata, por lo tanto, de una opción diferente a la aquí planteada, que está basada en ampliar las capacidades de un SIG preexistente.

Un ejemplo de las posibilidades de esta opción es el caso del programa SIG ARC/INFO, en este software se ha empezado a incluir procedimientos similares a los aquí tratados. Por ejemplo, ARC/INFO incluye algunos modelos de localización-asignación dentro de su versión más reciente: $1 .^{\circ}$. Para el sector privado: P-mediano. $2 .^{\circ}$. Para el sector público: P-mediano con restricción de máxima distancia, MEDIRES. Modelo DECLIN y Modelo DESNOLIN. $3 .^{\circ}$ Para los servicios de emergencia: Modelo COBEMAX y Modelo COBERES. En un intento de presentar una especie de Sistema de Ayuda a la Decisión, dispone de herramientas para probar varias alternativas, formular escenarios y realizar distintas simulaciones que puedan ayudar al decisor. De igual manera, existen herramientas para generar distintos gráficos (quizás demasiado simples) y poder así comparar las distintas alternativas encontradas.

Por otro lado, el programa LADDS (producido en el NCGIA de Estados Unidos) supone otro ejemplo interesante de relación entre varios de los modelos aquí mencionados y algunas capacidades de análisis semejantes a un SIG. En este caso tenemos la situación opuesta a la planteada en este artículo. LADDS es un conjunto de modelos de localización-asignación a los que se les añade unas mínimas posibilidades de tipo SIG. 
La elaboración de un sistema de ayuda a la decisión como el aquí planteado, por su mayor capacidad, entendemos que podría significar un interesante paso adelante en la resolución más correcta de este tipo de problemas.

\section{CONCLUSIONES}

La resolución de problemas espaciales acorde con las circunstancias tecnológicas de nuestro momento histórico demanda la disponibilidad de herramientas que, sustentadas en la informática y aprovechando los desarrollos en las técnicas de análisis de datos, posibiliten la adopción de decisiones sobre unas bases de conocimiento más fundado. Los sistemas de apoyo a la decisión, y dentro de ellos los de naturaleza espacial, conforman una prometedora línea de investigación y desarrollo en la que la Geografía debe profundizar en aras de aportar soluciones a necesidades de la sociedad. Con esa finalidad se ha esbozado, en este artículo, una propuesta de SADE orientado a mejorar la toma de decisiones sobre cuestiones de localización de equipamientos.

La principal conclusión es que existen los elementos para la elaboración de este tipo de herramientas: Sistemas de Información Geográfica flexibles y fáciles de ampliar, modelos matemáticos bien conocidos y facilidades de programación informática. Del mismo modo, la demanda de este tipo de instrumentos empieza a ser significativa. Por todo ello, parece necesario que se desarrollen con rapidez acciones en este sentido.

La participación de los geógrafos en este tipo de actividades abre, por otra parte, unas nuevas posibilidades de investigación y de conexión con las necesidades sociales que resultan de gran interés.

\section{BIBLIOGRAFÍA}

Armstong, M.; Densham, P., y Rushton, G. (1986): «Architecture for a micro-computer based decision support systems», Proceedings of the 2nd International Symposium on Spatial Data Handling, International Geographical Union, Williamsville, New York, pp. 120-131.

ARMSTONG, A. et al. (1992): «Cartographic Displays to Support Locational Decision Making», Cartography and GIS, vol. 19, n. ${ }^{\circ} 3$, pp. 154-164.

Barba Romero, S., y Pomerol, J.-C. (1997): Decisiones multicriterio. Fundamentos teóricos y utilización práctica, Alcalá de Henares, Servicio de publicaciones de la Universidad de Alcalá. 420 pp. 
BARREDO CANO, J. I. (1996): Evaluación multicriterio y Sistemas de Información Geográfica en la Ordenación del Territorio, Madrid, Editorial RA-MA, $264 \mathrm{pp}$.

Bloxham, C. A., y ChuRch, R. L. (1991): «The p-Median Scheduling and location problem», Papers in Regional Science, vol. 70, n. 1, pp. 21-36.

Bosque Sendra, J.; Chicharro Fernández, E.; Díaz Muñoz, M. A.; Escobar Martínez, F. J., y Galve MARTín A. (1992): «Datos perceptuales e individuales y el empleo de un SIG en el estudio de problemas sociales. Su aplicación en la localización de equipamientos urbanos», Actas del 1. er Congreso de la AESIGYT, Madrid, abril de 1992. Publicada en Co-

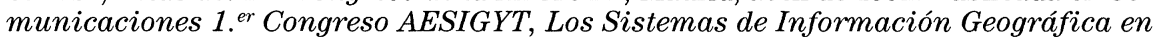
la gestión territorial, Madrid, AESIGYT, 392-409.

Bosque Sendra, J., y Franco MAAS, S. (1995): «Modelos de localización-asignación y evaluación multicriterio para la localización de instalaciones no deseables», Serie Geográfica, n. ${ }^{\circ}$ 5, pp. 97-114.

Bosque SENDRA, J., y MORENO JIMÉNEZ, A. (1990): «Facility location analysis and planning: a GIS approach», EGIS'90. First European Conference on Geographical Information Systems, Utrecht, EGIS Foundation, pp. 87-94.

Brocker, J. (1989): «How to Eliminate Certain Defects of the Potential Formula», Environment and Planning A., 21, 6, pp. 817-830.

CHURCH, R. L., y SORENSEN, P. (1996): «Integrating normative location models into GIS: problems and prospects with the p-median model», en P. LONGLEY y M. BATTY: Spatial Analysis: Modelling in a GIS environment, Cambridge, Geoinformation international, pp. 167-185.

DASKIn, M. S. (1995): Network and Discrete Location. Models, Algorithms and Applications, Nueva York, J. Wiley.

Densham, P. J. (1996): «Visual interactive locational analysis», en P. LONGLEY y M. BATTY: Spatial Analysis: Modelling in a GIS environment, Cambridge, Geoinformation international, pp. 185-206.

Densham, P. J. y RUSTHON, G. (1991): Designing and Implementing Strategies for Solving Large Location-Allocation Problems with Heuristic Methods, Santa Barbara, Ca., NCGIA, Technical Paper, 91-10, $37 \mathrm{p}$.

DENSHAM, P. J., y RUSTHON, G. (1992): «Strategies for Solving Large Location-Allocation Problems by Heuristic Methods», Environment anä Planning A, 24, p. 289-304.

ENACHE, M. (1994): «Integrating GIS with DSS: a research agenda», Urban and Regional Information Asociation, pp.154-166. (En http://wwwsgi.ursus.maine.edu/gisweb/spatdb/gis-lis/gi94009.html).

ENGLAND, J. R. et al. (1985): Information systems for policy planning in local government, Londres, Longman.

ER, M. (1988): «Decision support systems: A summary, problems and futur trends», Decision Support Systems, 4, p. 355-363.

ERKuT, E., y Neuman, S. (1988): «Analytical Models for Locating Undesirable Facilities», European Journal of Operational Research, vol. 40, (1988), pp. 275-291.

Goodchild, M. E., y NoronHA, V. T. (1983): Location-Allocation for Small Computers, Iowa, Department of Geography, University of Iowa.

Hillsman, E. L. (1984): «The P-Median Structure as an Unified Linear Model for LocationAllocation Analysis», Environment and Planning A, 16, 3, pp. 305-318.

HODGART, R. L. (1978): «Optimising access to public services: a review of problems, models and methods of locating central facilities», Progress in Human Geography, 2, 1, pp. $17-48$.

HuFF, D. L. (1964): «Defining and estimating a trading area», Journal of Marketing, 28, pp. 34-38.

JI, W., y JoHnston, J. (1994): «A GIS-Based decision support system for wetland permit analysis», GIS-LIS, pp.471-476. (En http://wwwsgi.ursus.maine.edu/gisweb/spatdb/gislis/gi94009.html). 
Jones, S. R. (1981): «Accesibility Measures: a literature Review», TRRL Laboratory, Report 967 , Crowthorne.

KOHSAKA, H. (1993): «A monitoring and locational decision support system for retail activity», Environment and Planning A, 25, p. 197-211.

MCALlister, D. M.: «Equity and Efficiency in Public Facility Location», Geographical Analysis, vol. 8, (1976), pp. 47-63.

MORENO JiMÉNEZ, A. (1995): «Planificación y gestión de servicios a la población desde la perspectiva territorial: algunas propuestas metodológicas», Boletín de la Asociación de Geógrafos Españoles, 20, 1995, p. 115-134.

MoReno JimÉnez, A., y ÁlVAREZ, C. (1997): «Análisis y evaluación de servicios públicos locales desde la perspectiva geográfica. Un estudio de caso", Gestión y Análisis de Políticas Públicas, 10, p. 85-99.

MoREno JiMÉnEz, A., y LÓPEZ DE LOS Mozos, M. A. (1989): «Organización espacial del sistema de centros de enseñanza general básica en el sureste de Madrid. Un análisis comparativo de modelos de localización-asignación», Revista de Educación, 290, pp. 407-442.

Moreno PÉREz, J.A. (1996): «Heurísticas de búsqueda para problemas discretos de localización-asignación», en Puerto Albandoz (editor), Lecturas en Teoría de la localización, Sevilla, Publicaciones de la Universidad de Sevilla, pp. 107-134.

MorRILL, R. L., y Symons, J.: «Efficiency and Equity aspects of Optimum Location», Geographical Analysis, vol. 9, (1977), pp.215-225.

MiÑoz Pérez, J. (1996): «Localización de centros no deseados», en Puerta Albandez (editor), Lecturas en Teoría de la localización, Sevilla, Publicaciones de la Universidad de Sevilla, pp. 135-146.

OBerg, S. (1976): «Methods of Describing Physical Access to Supply Points», Lund Studies in Geography, Ser. B, Human Geography, 43, 141 pp.

Pelegrín Pelegrín, B. (1996): «El problema general del p-centro en $\mathrm{R}^{\mathrm{N}}$ », en PUeRTo ALBANDOZ (ed.), Lecturas en Teoría de la localización, Sevilla, Publicaciones de la Universidad de Sevilla, pp. 149-166.

Puerto Albandoz, J. (ed., 1996): Lecturas en Teoría de la localización, Sevilla, Publicaciones de la Universidad de Sevilla, $236 \mathrm{pp}$.

RATICK, S. J., y WhITE, A. L. (1988): «A risk-sharing model for locating noxious facilities», Environment and Planning B, vol. 15, pp. 165-179.

TEITZ, M., y BART, P. (1968): «Heuristic methods for estimating the generalised vertex median of a weighted graph», Operations Research, 16, 5, pp. 955-961.

Resumen: Se describe el contenido y organización de un Sistema de Ayuda a la Decisión Espacial (SADE) (Spatial Decision Support System, SDSS), basado en un Sistema de Información Geográfica, diferentes modelos de localización-asignación y técnicas de evaluación multicriterio, que permita auxiliar a la determinación de la localización óptima de equipamientos sociales.

PALABRAS CLAVE: SIG, Modelos de localización-asignación, Evaluación Multicriterio, Sistema de Ayuda a la Decisión Espacial (SADE).

ABSTRACT: The content and organisation of a Spatial Decision Support System (SDSS) is described. The SDSS is based on a Geographical Information System (GIS), location-allocation models and techniques for evaluation multi-criteria, that allows to decide the optimal location of social facilities.

KEY wORDS: GIS, Location-Allocation Models, EMC, SDSS. 
RÉSUMÉ: On décrit le sujet et l'organisation d'un système d'aide á la décision spatial (Spatial Decision Support System, SDSS) à fin de prendre une décision (du point de vue spatial) obtenu á partir de: un Système d'Information Géographique (GIS), des differents modèles du localisation-assignation et du techniques d'Analyses Multicritère, qui permet nous aider à prendre une détermination la plus valable pour obtenir une localisation des équipaments sociaux.

Mots CLÉs: Systèmes d'information géographique, Analyse Multicritère, Systèmes d'aide á la décision. 